\title{
Factors associated with distress amongst a sample of Irish mothers at 4 months post-partum
}

\author{
A.E. Bennett and J.M. Kearney \\ School of Biological Sciences, Dublin Institute of Technology, Kevin Street, Dublin, Ireland
}

Carrying, and giving birth to, a child marks an important transition from one stage of a woman's life to another. While joy is so often associated with this time of a woman's life, it is also a time of increased vulnerability and psychological distress ${ }^{(1)}$. In light of the adverse consequences of distress on maternal and infant wellbeing, it is important to recognise factors which increase the likelihood of distress and to identify how these factors can be attenuated.

Mother-infant dyads were visited at home at 4 months post-partum by the lead researcher. Data on maternal sociodemographic and health behaviours were collected. Two validated instruments were used to assess maternal distress: the Mother and Baby Interaction Scale (MABISC) ${ }^{(2)}$ and the 14-item Resilience Scale ${ }^{(3)}$. Maternal perception of partner involvement during pregnancy was also assessed. Relationships between categorical variables were examined using cross-tabulations and Chi-squared tests, with significance being taken at $P<0.05$. Independent variables which were significant in these univariate analyses were included in multivariate analyses. Binary logistic regression was performed using the Forced Entry Method to predict distress in the post-partum period, where the dependent variable was based on scores from the MABISC. No significant distress was indicated by a score $\leq 7$, and significant distress was indicated by a score of $\geq 8$. Therefore, the codes for the dependent variable were: $1=$ distress and $0=n o$ distress. Statistical significance was taken at $p<0 \cdot 05$.

One hundred and seventy-two mothers provided data on their wellbeing at four months post-partum. The mean age of participating mothers was $31.6 \pm 4.9$ years. Similar to national feeding patterns in Ireland, over half $(55.9 \%, n 151)$ initiated breastfeeding, $44.8 \%$ ( $n 121)$ were doing any breastfeeding on discharge from hospital and 23.8 \% were doing any breastfeeding at 4 months post-partum. At 4 months post-partum, the majority $(64.0 \%, n 110)$ of mothers were coping well with the adjustment to motherhood. However, more than third $(36.0 \%, n 62)$ of mothers were experiencing some degree of distress at this time. Controlling for other factors, mothers were significantly $(p<0.05)$ more likely to be distressed at 4 months post-partum if they were breastfeeding (odds ratio (OR): 2.89 [95 \% confidence interval (CI): 1.29-6.47]); had low resilience (OR: 7.22 [95\% CI: 2.49-20.95]); or had a perception of suboptimal partner involvement during pregnancy (OR: $3 \cdot 89$ [95\% CI: 1.20-12.65]).

Women in Ireland who are breastfeeding at four months post-partum are in the minority, with only one in five Irish women doing any breastfeeding at this time ${ }^{(4)}$. The longstanding formula feeding culture in Ireland ${ }^{(5)}$ may increase the likelihood of breastfeeding mothers feeling distressed, particularly if the reality of the breastfeeding experience is not in line with maternal expectations. Therefore, it is important that women who express a positive antenatal intention to breastfeed are adequately prepared to breastfeed. It is also important that a mother's sense of resilience, or her ability to cope with the challenges of motherhood, is safeguarded at this time, with reassurance provided as needed. Maternal resilience can be bolstered by a mother's support network, most particularly her partner, and so it is important that expectant parents are given strategies on how to clearly communicate their support of one another as they enter parenthood for the first, or indeed subsequent, time.

1. Haga SM, Lynne A, Slinning K et al. (2012) Scand J Caring Sci 26, 458-466.

2. Hackney M, Braithwaite S \& Radcliffe G (1996) Health Visitor 69, 103-104.

3. Wagnild GM \& Young HM (1993) J Nurs Meas 1, 165-178.

4. Layte R \& McCrory C (2014) at: http://www.growingup.ie/fileadmin/user_upload/documents/Maternal_Health_Report/GUI_Infant_Maternal_ Health_4_web.pdf.

5. Brick A \& Nolan A (2014) Ir J Med Sci 183, 333-339. 